\title{
THE MUTUAL INDUCTANCE OF TWO SQUARE COILS*
}

\author{
BY
}

\section{T. H. GRONWALL}

In the design of apparatus for the absolute measurement of radio field intensity, it is necessary to compute the mutual inductance of two square coils in the following position: In a vertical plane, place two squares of sides $l$ and $L$, where $l<L$, so that their centers coincide and a pair of sides in each square are vertical. Rotate the square of side $l$ through an angle $\alpha$ about a vertical axis passing through the midpoints of the horizontal sides, and move the square of side $L$ through a distance $h$ perpendicularly to the original plane. We thus arrive at the arrangement presented in top and side views in the figure (p. 519).

The mutual inductance $I$ of two coils is given by the Neumann integral

$$
I=\iint \frac{\cos \theta}{r} d s d s^{\prime},
$$

where $d s$ and $d s^{\prime}$ are the line elements of the first and second coil, respectively, $\theta$ being the angle and $r$ the distance between $d s$ and $d s^{\prime}$.

In our case, the coils are made up from straight segments, and the integral may be expressed explicitly in terms of the elementary functions by means of formulas due to G. A. Campbell.t While these formulas are very interesting from a theoretical point of view, they do not work well in the present problem. In fact, $L$ and $l$ being given, the design of the apparatus requires the numerical values of $I$ for several values of $h$ and a large number of values of $\alpha$, and the formulas referred to would consequently have to be evaluated separately and independently for each combination of values of $h$ and $\alpha$. Even with the aid of the graphical method proposed by Campbell, this numerical work becomes rather formidable.

It is the purpose of the present paper to give a series expansion which solves the problem of numerical computation in what appears to be the simplest manner possible under the circumstances. The number of terms written out in the formula is sufficient to give $I / L$ with an error less than two units in the third decimal place under the condition $l<0.35 L$ occurring in practice (for $l=0.35 L$ and $h=\alpha=0$, this error is less than $0.15 \%$ ).

* Presented to the Society, April 28, 1923. This investigation was undertaken at the request of the American Telephone and Telegraph Company, and is published with their permission.

$\dagger$ G. A. Campbell, Mutual inductances of circuits composed of straight wires, Physical Review, new ser., vol. 5, pp. 452-458; June, 1915. 
The mutual inductance I of two square coils in the position shown in the figure is given by

where

$$
\frac{I}{L}=\sum_{n=1}^{\infty} \lambda^{2 n} \sum_{m=1}^{n} f_{m n}(x) \cos (2 m-1) \alpha,
$$

$$
x=\frac{2 h}{L}, \quad \lambda=\frac{l}{L},
$$

the series being convergent for $2 \lambda^{2}<1+x^{2}$. Retaining only terms up to $n=3$ inclusive, we obtain the approximate formula

$$
\begin{aligned}
\frac{I}{L}= & {\left[f_{11}(x) \lambda^{2}+f_{12}(x) \lambda^{4}+f_{13}(x) \lambda^{6}\right] \cos \alpha } \\
+ & {\left[f_{22}(x) \lambda^{4}+f_{23}(x) \lambda^{6}\right] \cos 3 \alpha } \\
+ & {\left[f_{33}(x) \lambda^{6}\right] \cos 5 \alpha }
\end{aligned}
$$

with an error less than 0.002 for $\lambda<0.35$. Writing

$$
a=\frac{1}{1+x^{2}}, \quad b=\frac{1}{\sqrt{2+x^{2}}},
$$

we have the following values of the coefficients:

$$
\begin{aligned}
& f_{11}(x)= 16 a b \\
& f_{12}(x)= 3 a^{2}\left[(-4+5 a)\left(b-\frac{2}{3} b^{3}+\frac{1}{5} b^{5}\right)+\frac{1}{3} b^{3}-\frac{1}{5} b^{5}\right], \\
& f_{22}(x)=\frac{5}{3} f_{12}(x), \\
& f_{13}(x)=7 a^{3}\left[\left(\frac{3}{4}-\frac{49}{12} a+\frac{21}{8} a^{2}\right)\left(b-\frac{4}{3} b^{3}+\frac{6}{5} b^{5}-\frac{4}{7} b^{7}+\frac{1}{9} b^{9}\right)\right. \\
& \quad+\left(\frac{13}{4}+\frac{77}{12} a\right)\left(\frac{1}{3} b^{3}-\frac{3}{5} b^{5}+\frac{3}{7} b^{7}-\frac{1}{9} b^{9}\right) \\
&\left.\quad-\frac{43}{8}\left(\frac{1}{5} b^{5}-\frac{2}{7} b^{7}+\frac{1}{9} b^{9}\right)\right], \\
& \quad+\left(\frac{31}{8}-\frac{245}{24} a+\frac{93}{16} a^{2}\right)\left(b-\frac{4}{3} b^{3}+\frac{6}{5} b^{5}-\frac{4}{7} b^{7}+\frac{1}{9} b^{9}\right) \\
& \quad+\left(-\frac{127}{8}+\frac{565}{24} a\right)\left(\frac{1}{3} b^{3}-\frac{3}{5} b^{5}+\frac{3}{7} b^{7}-\frac{1}{9} b^{9}\right) \\
&\left.\quad-\frac{19}{16}\left(\frac{1}{5} b^{5}-\frac{2}{7} b^{7}+\frac{1}{9} b^{9}\right)\right],
\end{aligned}
$$




$$
\begin{aligned}
f_{33}(x)=63 a^{3} & {\left[\left(\frac{3}{8}-\frac{11}{8} a+\frac{17}{16} a^{2}\right)\left(b-\frac{4}{3} b^{3}+\frac{6}{5} b^{5}-\frac{4}{7} b^{7}+\frac{1}{9} b^{9}\right)\right.} \\
& +\left(-\frac{3}{8}+\frac{3}{8} a\right)\left(\frac{1}{3} b^{3}-\frac{3}{5} b^{5}+\frac{3}{7} b^{7}-\frac{1}{9} b^{9}\right) \\
& \left.+\frac{1}{16}\left(\frac{1}{5} b^{5}-\frac{2}{7} b^{7}+\frac{1}{9} b^{9}\right)\right] .
\end{aligned}
$$

Proof

1. The Neumann integral. Consider first the vertical segments; letting $\overline{A C}$ be the horizontal distance between the two segments 2 and 6 , it is seen from the top view in the figure that

$$
\overline{A C^{2}}=\left(\frac{1}{2} L-\frac{1}{2} l \cos \alpha\right)^{2}+\left(h-\frac{1}{2} l \sin \alpha\right)^{2}
$$

Measure $x^{\prime}$ and $y^{\prime}$ along segments 2 and 6 , respectively, from their midpoints and positive in the direction of the arrow; then the distance between the two corresponding points is given by

$$
r_{26}^{2}=\left(\frac{1}{2} L-\frac{1}{2} l \cos c\right)^{2}+\left(h-\frac{1}{2} l \sin c\right)^{2}+\left(x^{\prime}-y^{\prime}\right)^{2} .
$$

Similarly

$$
\begin{aligned}
& r_{28}^{2}=\left(\frac{1}{2} L+\frac{1}{2} l \cos \alpha\right)^{2}+\left(h+\frac{1}{2} l \sin \alpha\right)^{2}+\left(x^{\prime}+y^{\prime}\right)^{2}, \\
& r_{46}^{2}=\left(\frac{1}{2} L+\frac{1}{2} l \cos \alpha\right)^{2}+\left(h-\frac{1}{2} l \sin \alpha\right)^{2}+\left(x^{\prime}+y^{\prime}\right)^{2}, \\
& r_{48}^{2}=\left(\frac{1}{2} L-\frac{1}{2} l \cos \alpha\right)^{2}+\left(h+\frac{1}{2} l \sin \alpha\right)^{2}+\left(x^{\prime}-y^{\prime}\right)^{2}
\end{aligned}
$$

Since $\theta=0$ for the segments 2,6 and 4,8 , but $\theta=\pi$ for the segments 2,8 and 4,6 , while $\theta=\pi / 2$ for a vertical and a horizontal segment, it follows that the contribution of the vertical segments to the Neumann integral is

$$
I^{\prime}=\int_{-\frac{1}{2} L}^{\frac{1}{2} L} d x^{\prime} \int_{-\frac{1}{2} l}^{\frac{1}{2} l} d y^{\prime}\left(\frac{1}{r_{26}}-\frac{1}{r_{28}}+\frac{1}{r_{48}}-\frac{1}{r_{46}}\right)
$$

Introducing the notations

$$
x=2 h / L, \quad \lambda=l / L
$$

and substituting $x^{\prime}=L x / 2, y^{\prime}=l y / 2=L \lambda y / 2$ in the integral, we obtain (2) $I^{\prime}=\frac{1}{2} L[F(\alpha, \varkappa, \lambda)+F(\alpha,-x, \lambda)+F(\alpha, x,-\lambda)+F(\alpha,-x,-\lambda)]$, 
where

and

(4)

$$
F(\alpha, x, \lambda)=\int_{-1}^{1} \int_{-1}^{1} \frac{\lambda}{V} d x d y
$$

$$
V^{2}=(1-\lambda \cos \alpha)^{2}+(x-\lambda \sin \alpha)^{2}+(x-\lambda y)^{2} \text {. }
$$
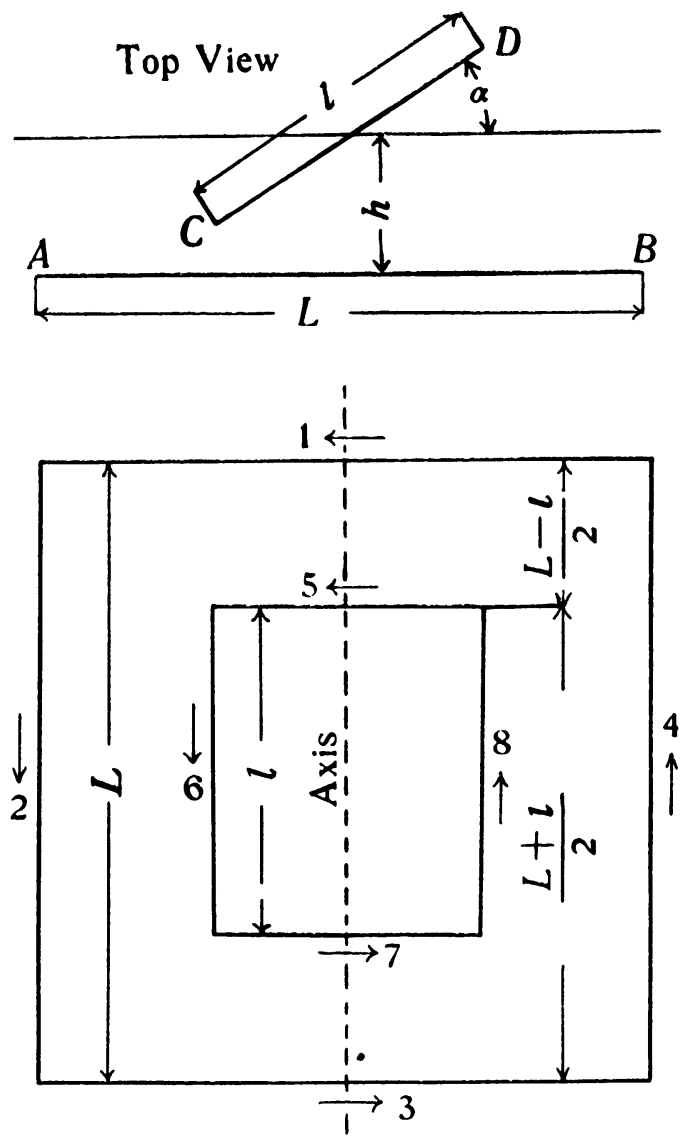

Side View

Turning our attention to the horizontal segments, let us measure $x^{\prime}$ and $y^{\prime}$ along segments 1 and 5, respectively, from their midpoints and positive in the direction of the arrow; it is then seen from the figure that the distance $r_{15}$ between the two corresponding points is given by

$$
r_{15}^{2}=\left(\frac{1}{2} L-\frac{1}{2} l\right)^{2}+\left(x^{\prime}-y^{\prime} \cos \alpha\right)^{2}+\left(h-y^{\prime} \sin \alpha\right)^{2},
$$

and similarly

$$
\begin{aligned}
& r_{17}^{2}=\left(\frac{1}{2} L+\frac{1}{2} l\right)^{2}+\left(x^{\prime}+y^{\prime} \cos \alpha\right)^{2}+\left(h+y^{\prime} \sin \alpha\right)^{2}, \\
& r_{35}^{2}=r_{17}^{2}, \quad r_{37}^{2}=r_{15}^{2} .
\end{aligned}
$$


Since $\theta=\alpha$ for the segments 1,5 and 3,7 , but $\theta=\pi+\alpha$ for the segments 1,7 and 3,5 , it follows that the contribution of the horizontal segments to the Neumann integral is

$$
I^{\prime \prime}=\int_{-\frac{1}{2} L}^{\frac{1}{2} L} d x^{\prime} \int_{-\frac{1}{2} l}^{\frac{1}{2} l} d y^{\prime}\left(\frac{2 \cos \alpha}{r_{15}}-\frac{2 \cos \alpha}{r_{17}}\right) .
$$

By the same substitution as before, we obtain

where

$$
I^{\prime \prime}=L[G(\kappa, x, \lambda)+G(\kappa, x,-\lambda)]
$$

$$
G(\kappa, x, \lambda)=\int_{-1}^{1} \int_{-1}^{1} \frac{\lambda \cos \alpha}{H} d x d y
$$

and

(6)

$$
H^{2}=(1-\lambda)^{2}+(x-\lambda y \cos \alpha)^{2}+(x-\lambda y \sin \alpha)^{2}
$$

We have $G(\alpha,-x, \lambda)=G(\alpha, x, \lambda)$, as is seen from (5) and (6) upon changing $x, y$ and $x$ into $-x,-y$ and $-x$ respectively. Thus the formula for $I^{\prime \prime}$ may be written in a form exactly similar to (2):

(7) $I^{\prime \prime}=\frac{1}{2} L[G(\alpha, x, \lambda)+G(\alpha,-x, \lambda)+G(\alpha, x,-\lambda)+G(\alpha,-x,-\lambda)]$.

2. Expansion in powers of $\lambda$. From (4) and (6), we have

$$
\begin{aligned}
& V^{2}=1+x^{2}+x^{2}-2(\cos \alpha+x \sin \alpha+x y) \lambda+\left(1+y^{2}\right) \lambda^{2}, \\
& H^{2}=1+x^{2}+x^{2}-2(1+x y \cos \alpha+x y \sin \alpha) \lambda+\left(1+y^{2}\right) \lambda^{2},
\end{aligned}
$$

and consequently

$$
\frac{1}{V}=\left(1+x^{2}+x^{2}\right)^{-1 / 2}\left(1-2 \xi z+z^{2}\right)^{-1 / 2},
$$

$$
\frac{1}{H}=\left(1+x^{2}+x^{2}\right)^{-1 / 2}\left(1-2 \eta z+z^{2}\right)^{-1 / 2},
$$

where

$$
\begin{aligned}
& \xi=\frac{\cos \alpha+x \sin \alpha+x y}{\left(1+x^{2}+x^{2}\right)^{1 / 2}\left(1+y^{2}\right)^{1 / 2}}, \\
& \eta=\frac{1+x y \cos \alpha+x y \sin \alpha}{\left(1+x^{2}+x^{2}\right)^{1 / 2}\left(1+y^{2}\right)^{1 / 2}}, \\
& z=\lambda\left(\frac{1+y^{2}}{1+x^{2}+x^{2}}\right)^{1 / 2} .
\end{aligned}
$$


We now note the relations

$$
\begin{aligned}
& \left(1+x^{2}+x^{2}\right)\left(1+y^{2}\right)-(\cos \alpha+x \sin \alpha+x y)^{2} \\
& =(\sin \alpha-x \cos \alpha)^{2}+(x \cos \alpha-y)^{2}+(x \sin \alpha-x y)^{2} \geqq 0, \\
& \left(1+x^{2}+x^{2}\right)\left(1+y^{2}\right)-(1+x y \cos \alpha+x y \sin \alpha)^{2} \\
& =(x y \sin \alpha-x y \cos \alpha)^{2}+(x-y \sin \alpha)^{2}+(x-y \cos \alpha)^{2} \geqq 0,
\end{aligned}
$$

from which it follows that $\xi^{2} \leqq 1, \eta^{2} \leqq 1$, or

$$
-1 \leqq \xi \leqq 1, \quad-1 \leqq \eta \leqq 1
$$

From the last of (9), it is seen that, for $-1 \leqq x \leqq 1,-1 \leqq y \leqq 1$, and for

$$
2 \lambda^{2}<1+x^{2}
$$

we have

$$
|z|<|\lambda|\left(\frac{2}{1+x^{2}}\right)^{1 / 2}<1
$$

and in consequence of (10), (11) and (12), the expansions

$$
\begin{aligned}
& \left(1-2 \xi z+z^{2}\right)^{-1 / 2}=\sum_{n=0}^{\infty} P_{n}(\xi) z^{n}, \\
& \left(1-2 \eta z+z^{2}\right)^{-1 / 2}=\sum_{n=0}^{\infty} P_{n}(\eta) z^{n},
\end{aligned}
$$

where $P_{n}$ are the Legendre polynomials, converge uniformly for $-1 \leqq x \leqq 1$, $-1 \leqq y \leqq 1$. Consequently, we may introduce these expansions in the formulas for $F(\alpha, x, \lambda)$ and $G(\alpha, x, \lambda)$ and integrate term by term, thus obtaining

$$
\begin{aligned}
& F(\alpha, x, \lambda)=\sum_{n=0}^{\infty} \int_{-1}^{1} \int_{-1}^{1} \frac{\lambda P_{n}(\xi) z^{n}}{\left(1+x^{2}+x^{2}\right)^{1 / 2}} d x d y \\
& G(\alpha, x, \lambda)=\sum_{n=0}^{\infty} \int_{-1}^{1} \int_{-1}^{1} \frac{\lambda \cos \alpha P_{n}(\eta) z^{n}}{\left(1+x^{2}+x^{2}\right)^{1 / 2}} d x d y .
\end{aligned}
$$

Changing $\lambda$ into $-\lambda$, it is seen from (9) that $z$ changes into $-z$, while $\xi$ and $\eta$ remain the same, being independent of $\lambda$. Consequently

$$
F(\alpha, \varkappa, \lambda)+F(\alpha, \varkappa,-\lambda)=\sum_{n=0}^{\infty} \int_{-1}^{1} \int_{-1}^{1} \frac{\left[1+(-1)^{n}\right] \lambda P_{n}(\xi) z^{n}}{\left(1+x^{2}+x^{2}\right)^{1 / 2}} d x d y
$$


with a similar formula for $G$, or

$$
\begin{aligned}
& F(\alpha, x, \lambda)+F(\alpha, x,-\lambda)=2 \sum_{n=0}^{\infty} \int_{-1}^{1} \int_{-1}^{1} \frac{\lambda P_{2 n+1}(\xi) z^{2 n+1}}{\left(1+x^{2}+x^{2}\right)^{1 / 2}} d x d y \\
& G(\alpha, x, \lambda)+G(\alpha, x,-\lambda)=2 \sum_{n=0}^{\infty} \int_{-1}^{1} \int_{-1}^{1} \frac{\lambda \cos \alpha P_{2 n+1}(\eta) z^{2 n+1}}{\left(1+x^{2}+x^{2}\right)^{1 / 2}} d x d y .
\end{aligned}
$$

Consequently, by (2) and (5),

$$
\frac{I}{L}=\frac{I^{\prime}}{L}+\frac{I^{\prime \prime}}{L}=\sum_{n=1}^{\infty} \varphi_{n}(\alpha, \varkappa) \lambda^{2 n}
$$

where

(14) $\varphi_{n}(\alpha, x)$

$=\int_{-1}^{1} \int_{-1}^{1} \frac{\left[P_{2 n-1}(\xi)+P_{2 n-1}\left(\xi_{1}\right)+\cos \alpha P_{2 n-1}(\eta)+\cos \alpha P_{2 n-1}\left(\eta_{1}\right)\right]\left(1+y^{2}\right)^{n-1 / 2}}{\left(1+x^{2}+x^{2}\right)^{n}} d x d y$

and $\xi_{1}$ and $\eta_{1}$ are obtained from $\xi$ and $\eta$ in (9) by changing $x$ into $-x$, the series (13) being convergent under the condition (11).

3. Upper bound for the absolute value of the remainder in the series. Before evaluating the expression (14), it is appropriate to form an estimate of the error $R_{p}$ committed by retaining in (13) only the first $p$ terms, that is, only terms containing powers of $\lambda$ up to the $2 p$ th inclusive. This error is evidently the series remainder

so that

$$
R_{p}=\sum_{n=p}^{\infty} \varphi_{n+1}(\alpha, x) \lambda^{2 n+2},
$$

$$
\left|R_{p}\right| \leqq \sum_{n=p}^{\infty}\left|\varphi_{n+1}(\alpha, \varkappa)\right| \lambda^{2 n+2}
$$

Now $\xi, \xi_{1}, \eta$ and $\eta_{1}$ all lie in the interval from -1 to +1 by (10), and consequently every Legendre polynomial in one of these four arguments does not exceed unity in absolute value, so that (14) gives

$$
\left|\varphi_{n+1}(\alpha, x)\right| \leqq 2(1+|\cos \alpha|) \int_{-1}^{1} \int_{-1}^{1} \frac{\left(1+y^{2}\right)^{n+1 / 2}}{\left(1+x^{2}+x^{2}\right)^{n+1}} d x d y
$$

To get an upper bound for the expression to the right in (17), we observe that

$$
\begin{aligned}
\int_{-1}^{1} \frac{d x}{\left(1+x^{2}+x^{2}\right)^{n+1}} & <\int_{-\infty}^{\infty} \frac{d x}{\left(1+x^{2}+x^{2}\right)^{n+1}} \\
& =\frac{1 \cdot 3 \cdot 5 \cdots(2 n-1)}{2 \cdot 4 \cdot 6 \cdots 2 n} \cdot \frac{\pi}{\left(1+x^{2}\right)^{n+1 / 2}}
\end{aligned}
$$


the last integral being obtained by differentiating the integral

$$
\int_{-\infty}^{\infty} \frac{d x}{1+x^{2}+x^{2}}=\left[\frac{1}{\left(1+x^{2}\right)^{1 / 2}} \arctan \frac{x}{\left(1+x^{2}\right)^{1 / 2}}\right]_{-\infty}^{\infty}=\frac{\pi}{\left(1+x^{2}\right)^{1 / 2}}
$$

$n$ times in respect to $x^{2}$. Consequently we have

(18) $\int_{-1}^{1} \frac{d x}{\left(1+x^{2}+x^{2}\right)^{n+1}}<\frac{1 \cdot 3 \cdot 5 \cdots(2 p-1)}{2 \cdot 4 \cdot 6 \cdots 2 p} \cdot \frac{\pi}{\left(1+x^{2}\right)^{n+1 / 2}}$ for $n \geqq p$.

We also need a sufficiently close upper bound for $\int_{-1}^{1}\left(1+y^{2}\right)^{n+1 / 2} d y$; the identity

$$
\frac{d\left[y\left(1+y^{2}\right)^{n+1 / 2}\right]}{d y}=(2 n+2)\left(1+y^{2}\right)^{n+1 / 2}-(2 n+1)\left(1+y^{2}\right)^{n-1 / 2}
$$

yields the reduction formula

$$
\int_{-1}^{1}\left(1+y^{2}\right)^{n+1 / 2} d y=\frac{2^{n} \sqrt{2}}{n+1}+\frac{2 n+1}{2 n+2} \int_{-1}^{1}\left(1+y^{2}\right)^{n-1 / 2} d y
$$

from which we readily obtain

$$
\int_{-1}^{1}\left(1+y^{2}\right)^{4+1 / 2} d y=\frac{4607 \sqrt{2}}{640}+\frac{63}{256} \log (1+\sqrt{2})=10.397 .
$$

It will now be shown that

$$
\int_{-1}^{1}\left(1+y^{2}\right)^{n+1 / 2} d y<\frac{2^{n+1} \cdot 3 \sqrt{2}}{3 n+1} \text { for } n \geqq 4 \text {. }
$$

In fact, for $n=4$ the expression to the right equals 10.443, so that (20) holds in this case, and further numerical computation by means of (19) shows that the inequality is verified also for $n=5,6$ and 7 . Now assume (20) to be true for the exponent $n-1$; then (19) gives

$$
\int_{-1}^{1}\left(1+y^{2}\right)^{n+1 / 2} d y<\frac{2^{n} \sqrt{2}}{n+1}+\frac{2 n+1}{2 n+2} \cdot \frac{2^{n} \cdot 3 \sqrt{2}}{3 n-2}
$$

and consequently

$$
\begin{array}{rl}
\int_{-1}^{1}\left(1+y^{2}\right)^{n+1 / 2} & d y-\frac{2^{n+1} \cdot 3 \sqrt{2}}{3 n+1} \\
& <2^{n-1} \sqrt{2}\left[\frac{2}{n+1}+\frac{3(2 n+1)}{(n+1)(3 n-2)}-\frac{12}{3 n+1}\right] \\
& =\frac{2^{n-1} \sqrt{2}(23-3 n)}{(n+1)(3 n-2)(3 n+1)}<0 \text { for } n \geqq 8
\end{array}
$$

so that (20) is true for all $n \geqq 8$, which completes the proof. 
Introducing (18) and (20) in (17), it follows from (16) that

$$
\left|R_{p}\right|<2(1+|\cos \alpha|) \frac{1 \cdot 3 \cdot 5 \cdots(2 p-1) \cdot 3 \pi \sqrt{2}}{2 \cdot 4 \cdot 6 \cdots 2 p} \sum_{n=p}^{\infty} \frac{1}{3 n+1} \frac{2^{n+1} \lambda^{2 n+2}}{\left(1+x^{2}\right)^{n+1 / 2}}
$$

or replacing $3 n+1$ by $3 p+1$, which increases each term in the series except the first, and summing the resulting geometric series,

$$
\left|R_{p}\right|<(1+|\cos c|) \frac{1 \cdot 3 \cdot 5 \cdots(2 p-1)}{2 \cdot 4 \cdot 6 \cdots 2 p} \cdot \frac{6 \pi \sqrt{2\left(1+x^{2}\right)}}{3 p+1} \cdot \frac{\left(\frac{2 \lambda^{2}}{1+x^{2}}\right)^{p+1}}{1-\frac{2 \lambda^{2}}{1+x^{2}}}
$$

which is valid for $p \geqq 4$ and constitutes the desired upper bound for the absolute value of the error committed in neglecting all powers of $\lambda$ higher than the $2 p$ th.

It is evident from (21) that the right hand member increases with $|\cos \alpha|$, but decreases as $x$ increases. Making $\alpha=0, x=0$ and $p=4$, we therefore have

$$
\left|R_{4}\right|<2 \cdot \frac{1 \cdot 3 \cdot 5 \cdot 7}{2 \cdot 4 \cdot 6 \cdot 8} \cdot \frac{6 \pi \sqrt{2}}{13} \cdot \frac{\left(2 \lambda^{2}\right)^{5}}{1-2 \lambda^{2}}=1.122 \cdot \frac{\left(2 \lambda^{2}\right)^{5}}{1-2 \lambda^{2}}
$$

for all values of $\alpha$ and $x$.

For purposes of comparison, the exact values of $I / L$ and $R_{4}$ were computed from (65) for $\alpha=0, x=0$ and $\lambda=0.30,0.35$ and 0.40 , as well as the upper bound of $\left|R_{4}\right|$ from (22), with the following results:

$\begin{array}{cccc}\lambda & I / L & R_{4} \text { (exact) } & \left|R_{4}\right| \text { by }(22) \\ 0.30 & 1.05870 & +0.00001 & <0.00026 \\ 0.35 & 1.46258 & +0.00005 & <0.00131 \\ 0.40 & 1.94440 & +0.00021 & <0.00553\end{array}$

4. Expansion in cosines of the odd multiples of $\alpha$. We shall now expand each $\varphi_{n}(\alpha, x)$ as given by (14) in a finite Fourier series of the form

$$
\varphi_{n}(\alpha, x)=\sum_{m=1}^{n} f_{m n}(x) \cos (2 m-1) \alpha
$$

To this purpose, we introduce the associated Legendre polynomials

$$
P_{n}^{m}(z)=\left(1-z^{2}\right)^{m / 2} \cdot \frac{d^{m} P_{n}(z)}{d z^{m}}
$$


with their representation by an integral of the Laplace type

$$
P_{n}^{m}(z)=\frac{(n+m) !}{n ! 2 \pi i^{m}} \int_{-\pi}^{\pi}\left(z+i \sqrt{1-z^{2}}\right)^{n} \cos m \varphi d \varphi
$$

and finally the addition theorem for the Legendre polynomials

$$
\begin{array}{r}
P_{n}\left(\cos \theta \cos \theta^{\prime}+\sin \theta \sin \theta^{\prime} \cos \omega\right)=P_{n}(\cos \theta) P_{n}\left(\cos \theta^{\prime}\right) \\
+2 \sum_{m=1}^{n} \frac{(n-m) !}{(n+m) !} P_{n}^{n 2}(\cos \theta) P_{n}^{m}\left(\cos \theta^{\prime}\right) \cos m \omega
\end{array}
$$

where $0 \leqq \theta \leqq \pi$ and $0 \leqq \boldsymbol{\theta}^{\prime} \leqq \pi$. $^{*}$

Defining $\beta$ by the equations

$$
\cos \beta=\frac{1}{\left(1+x^{2}\right)^{1 / 2}}, \quad \quad \sin \beta=\frac{x}{\left(1+x^{2}\right)^{1 / 2}},
$$

and making

$$
\begin{array}{ll}
\cos \theta=\frac{x}{\left(1+x^{2}+x^{2}\right)^{1 / 2}}, & \sin \theta=\frac{\left(1+x^{2}\right)^{1 / 2}}{\left(1+x^{2}+x^{2}\right)^{1 / 2}}, \\
\cos \theta^{\prime}=\frac{y}{\left(1+y^{2}\right)^{1 / 2}}, & \sin \theta^{\prime}=\frac{1}{\left(1+y^{2}\right)^{1 / 2}},
\end{array}
$$

we find from (9) that

$$
\begin{aligned}
& \xi=\cos \theta \cos \theta^{\prime}+\sin \theta \sin \theta^{\prime} \cos (\alpha-\beta), \\
& \xi_{1}=\cos \theta \cos \theta^{\prime}+\sin \theta \sin \theta^{\prime} \cos (\alpha+\beta)
\end{aligned}
$$

using also the formula $\cos (\alpha-\beta)+\cos (\alpha+\beta)=2 \cos \alpha \cos \beta$, we obtain from (26)

$$
\begin{aligned}
P_{2 n-1}(\xi) & +P_{2 n-1}\left(\xi_{1}\right)=2 P_{2 n-1}(\cos \theta) P_{2 n-1}\left(\cos \theta^{\prime}\right) \\
& +4 \sum_{m=1}^{2 n-1}\left[\frac{(2 n-1-m) !}{(2 n-1+m) !} P_{2 n-1}^{m}(\cos \theta) P_{2 n-1}^{m}\left(\cos \theta^{\prime}\right) \cos m \beta \cos m \alpha\right] .
\end{aligned}
$$

Changing $y$ into $-y$, it is seen that

$$
\int_{-1}^{1} P_{2 n-1}^{m}\left(\cos \theta^{\prime}\right)\left(1+y^{2}\right)^{n-1 / 2} d y=\int_{-1}^{1} P_{2 n-1}^{m}\left(-\cos \theta^{\prime}\right)\left(1+y^{2}\right)^{n-1 / 2} d y
$$

* For these formulas, see for instance Whittaker and Watson, Modern Analysis, 2d edition, pp. 317-322. It should be noted that in establishing (25) and (26), these authors use a function $P_{n}^{m}$ which is the one in (24) multiplied by $i^{m}$. 
and by $(24), P_{2 n-1}^{m}(-z)=(-1)^{2 n-1-m} P_{2 n-1}^{m}(z)$, so that the integral vanishes when $m$ is even; consequently

$$
\begin{aligned}
\int_{-1}^{1} \int_{-1}^{1} \frac{\left[P_{2 n-1}(\xi)+P_{2 n-1}\left(\xi_{1}\right)\right]\left(1+y^{2}\right)^{n-1 / 2}}{\left(1+x^{2}+x^{2}\right)^{n}} d x d y \\
=\sum_{m=1}^{n} g_{m n}(x) \cos (2 m-1) \alpha
\end{aligned}
$$

where

$$
\begin{aligned}
& g_{m n}(x)=\frac{4 \cdot(2 n-2 m) !}{(2 n+2 m-2) !} \cos (2 m-1) \beta \\
& \times \int_{-1}^{1} P_{2 n-1}^{2 m-1}\left(\frac{x}{\left(1+x^{2}+x^{2}\right)^{1 / 2}}\right) \frac{d x}{\left(1+x^{2}+x^{2}\right)^{n}} \\
& \times \int_{-1}^{1} P_{2 n-1}^{2 m-1}\left(\frac{y}{\left(1+y^{2}\right)^{1 / 2}}\right)\left(1+y^{2}\right)^{n-1 / 2} d y .
\end{aligned}
$$

Before proceeding further, we shall evaluate the integral

$$
\int_{-\pi}^{\pi}(\cos \varphi)^{a} \cos b \varphi d \varphi
$$

where $a$ and $b$ are positive integers or zero. Since the corresponding integral with $\sin b \varphi$ instead of $\cos b \varphi$ vanishes, the integrand being odd, our integral is equal to

$$
\begin{aligned}
\int_{-\pi}^{\pi}(\cos \varphi)^{a} e^{b \varphi i} d \varphi & =\int_{-\pi}^{\pi}\left(\frac{e^{p i}+e^{-\varphi i}}{2}\right)^{a} e^{b \varphi i} d \varphi \\
& =\sum_{p=0}^{a}\left[\frac{a !}{2^{a} p !(a-p) !} \int_{-\pi}^{\pi} e^{(2 p-a+b) \varphi i} d \varphi\right]
\end{aligned}
$$

and in the last expression, every integral vanishes for which $2 p-a+b \neq 0$. Consequently

$$
\begin{aligned}
\int_{-\pi}^{\pi}(\cos \varphi)^{a} \cos b \varphi d \varphi & =\frac{\pi}{2^{a-1}} \cdot \frac{a !}{((a-b) / 2) !((a+b) / 2) !} \\
& =0 \text { in all other cases. }
\end{aligned}
$$

The last factor in (30) is now readily evaluated by means of (25), which gives 


$$
\begin{aligned}
A_{m n} & =\int_{-1}^{1} P_{2 n-1}^{2 m-1}\left(\frac{y}{\left(1+y^{2}\right)^{1 / 2}}\right)\left(1+y^{2}\right)^{n-1 / 2} d y \\
& =\frac{(2 n+2 m-2) !}{(2 n-1) ! 2 \pi} i^{2 n-1} \int_{-1}^{1} d y \int_{-\pi}^{\pi}(y+i \cos \varphi)^{2 n-1} \cos (2 m-1) \varphi d \varphi .
\end{aligned}
$$

Expanding $(y+i \cos \varphi)^{2 n-1}$ by the binomial theorem and using (31), we readily find

$$
A_{m n}=\sum_{p=m}^{n} \frac{(-1)^{p-m}(2 n+2 m-2) !}{2^{2 p-2}(p-m) !(p+m-1) !(2 n-2 p+1) !} .
$$

Incidentally it may be noted that, if we integrate first with respect to $y$ in the double integral in (32), and then apply (25), we obtain

$$
A_{m n}=\frac{2^{n+1}}{2 n+2 m-1} P_{2 n}^{2 m-1}\left(\frac{1}{\sqrt{2}}\right) .
$$

In the remaining integral in (30), we introduce the $\theta$ given by (28) as integration variable and obtain by (25)

$$
\begin{aligned}
& \int_{-1}^{1} P_{2 n-1}^{2 m-1}\left(\frac{x}{\left(1+x^{2}+x^{2}\right)^{1 / 2}}\right) \frac{d x}{\left(1+x^{2}+x^{2}\right)^{n}} \\
& \begin{aligned}
(34)= & \int_{\gamma}^{\pi-\gamma} P_{2 n-1}^{2 m-1}(\cos \theta)(\cos \beta)^{2 n-1}(\sin \theta)^{2 n-2} d \theta \\
= & \frac{(2 n+2 m-2) !(\cos \beta)^{2 n-1}}{(2 n-1) ! 2 \pi i^{2 m-1}} \int_{\gamma}^{\pi-\gamma}(\sin \theta)^{2 n-2} d \theta \\
& \times \int_{-\pi}^{\pi}(\cos \theta+i \sin \theta \cos \varphi)^{2 n-1} \cos (2 m-1) \varphi d \varphi
\end{aligned}
\end{aligned}
$$

where $\gamma$ is determined by

so that

$$
\cos \gamma=\frac{1}{\left(2+x^{2}\right)^{1 / 2}}, \quad \sin \gamma=\frac{\left(1+x^{2}\right)^{1 / 2}}{\left(2+x^{2}\right)^{1 / 2}},
$$

Expanding by the binomial theorem under the last integration sign in (34) and using (31), it is seen that

$$
\begin{aligned}
& \int_{-1}^{1} P_{2 n-1}^{2 m-1}\left(\frac{x}{\left(1+x^{2}+x^{2}\right)^{1 / 2}}\right) \frac{d x}{\left(1+x^{2}+x^{2}\right)^{n}} \\
& \quad=(\cos \beta)^{2 n-1} \sum_{p=0}^{n-m}\left(\frac{(-1)^{n-m-p}(2 n+2 m-2) !}{2^{2 n-2 p-2}(2 p) !(n-m-p) !(n+m-p-1) !} \psi_{n p}(\gamma)\right)
\end{aligned}
$$


where

$$
\begin{aligned}
\psi_{n p}(\gamma) & =\frac{1}{2} \int_{\gamma}^{\pi-\gamma}(\cos \theta)^{2 p}(\sin \theta)^{4 n-2 p-3} d \theta \\
& =\frac{1}{2} \int_{\gamma}^{\pi-\gamma}(\cos \theta)^{2 p}\left(1-\cos ^{2} \theta\right)^{2 n-p-2} d \cos \theta
\end{aligned}
$$

or expanding by the binomial theorem and integrating

$$
\psi_{n p}(\gamma)=\sum_{s=0}^{2 n-p-2}\left(\frac{(-1)^{s}(2 n-p-2) !}{s !(2 n-p-s-2) !} \cdot \frac{(\cos \gamma)^{2 p+2 s+1}}{2 p+2 s+1}\right)
$$

From (30), (32), (37) and (38) it now follows that

$$
\begin{aligned}
g_{m n}(x)= & 16 A_{m n} \cos (2 m-1) \beta(\cos \beta)^{2 n-1} \\
& \times \sum_{p=0}^{n-m}\left(\frac{(-1)^{n-m-p}(2 n-2 m) !}{2^{2 n-2 p}(2 p) !(n-m-p) !(n+m-p-1) !} \psi_{n p}(\gamma)\right) .
\end{aligned}
$$

To deal in a similar manner with the terms in (14) which contain $\eta$ and $\eta_{1}$, we make

$$
\begin{array}{ll}
\cos \tau=\frac{x}{\left(x^{2}+x^{2}\right)^{1 / 2}}, & \sin \tau=\frac{x}{\left(x^{2}+x^{2}\right)^{1 / 2}} \\
\cos \theta_{1}=\frac{1}{\left(1+x^{2}+x^{2}\right)^{1 / 2}}, & \sin \theta_{1}=\frac{\left(x^{2}+x^{2}\right)^{1 / 2}}{\left(1+x^{2}+x^{2}\right)^{1 / 2}} \\
\cos \theta_{1}^{\prime}=\frac{1}{\left(1+y^{2}\right)^{1 / 2}}, & \sin \theta_{1}^{\prime}=\frac{|y|}{\left(1+y^{2}\right)^{1 / 2}}
\end{array}
$$

It now follows from (9) that for $y>0$

while for $y<0$

$$
\begin{aligned}
& \eta=\cos \theta_{1} \cos \theta_{1}^{\prime}+\sin \theta_{1} \sin \theta_{1}^{\prime} \cos (\alpha-\tau), \\
& \eta_{1}=\cos \theta_{1} \cos \theta_{1}^{\prime}+\sin \theta_{1} \sin \theta_{1}^{\prime} \cos (\alpha+\tau),
\end{aligned}
$$

$$
\begin{aligned}
& \eta=\cos \theta_{1} \cos \theta_{1}^{\prime}+\sin \theta_{1} \sin \theta_{1}^{\prime} \cos (\alpha-\tau+\pi), \\
& \eta_{1}=\cos \theta_{1} \cos \theta_{1}^{\prime}+\sin \theta_{1} \sin \theta_{1}^{\prime} \cos (\alpha+\tau+\pi) .
\end{aligned}
$$

From (26), it is now seen that 


$$
\begin{aligned}
\int_{-1}^{1} P_{2 n-1}(\eta)\left(1+y^{2}\right)^{n-1 / 2} d y=P_{2 n-1}\left(\cos \theta_{1}\right) \int_{-1}^{1} P_{2 n-1}\left(\cos \theta_{1}^{\prime}\right)\left(1+y^{2}\right)^{n-1 / 2} d y \\
+2 \sum_{n=1}^{2 n-1}\left\{\frac{(2 n-1-m) !}{(2 n-1+m) !} P_{2 n-1}^{m}\left(\cos \theta_{1}\right) \cos m(\alpha-\tau)\right. \\
\times\left[\int_{0}^{1} P_{2 n-1}^{m}\left(\cos \theta_{1}^{\prime}\right)\left(1+y^{2}\right)^{n-1 / 2} d y\right. \\
\left.\left.+(-1)^{m} \int_{-1}^{\infty} P_{2 n-1}^{m}\left(\cos \theta_{1}^{\prime}\right)\left(1+y^{2}\right)^{n-1 / 2} d y\right]\right\},
\end{aligned}
$$

and since the integrand in the last two integrals is even,

$$
\begin{aligned}
& \int_{-1}^{1} P_{2 n-1}(\eta)\left(1+y^{2}\right)^{n-1 / 2} d y=B_{0 n} P_{2 n-1}\left(\cos \theta_{1}\right) \\
& \quad+2 \sum_{m=1}^{n-1}\left[\frac{(2 n-2 m-1) !}{(2 n+2 m-1) !} B_{m n} P_{2 n-1}^{2 m}\left(\cos \theta_{1}\right) \cos 2 m(\alpha-\tau)\right]
\end{aligned}
$$

where

$$
\begin{aligned}
B_{m n} & =\int_{-1}^{1} P_{2 n-1}^{2 m}\left(\frac{1}{\left(1+y^{2}\right)^{1 / 2}}\right)\left(1+y^{2}\right)^{n-1 / 2} d y \\
& =\frac{(2 n+2 m-1) !}{(2 n-1) ! 2 \pi i^{2 m}} \int_{-1}^{1} d y \int_{-\pi}^{\pi}(1+i y \cos \varphi)^{2 n-1} \cos 2 m \varphi d \varphi,
\end{aligned}
$$

or expanding by the binomial theorem and applying (31),

$$
B_{m n}=\sum_{p=m}^{n-1}\left(\frac{(-1)^{p-m}(2 n+2 m-1) !}{(2 p+1)} \frac{2^{2 p-1}(p-m) !(p+m) !(2 n-2 p-1) !}{)}\right) .
$$

By the aid of the formula $\cos (\alpha-\beta)+\cos (\alpha+\beta)=2 \cos \alpha \cos \beta$, (42) gives

where

$$
\begin{gathered}
\int_{-1}^{1} \int_{-1}^{1} \frac{\left[P_{2 n-1}(\eta)+P_{2 n-1}\left(\eta_{1}\right)\right]\left(1+y^{2}\right)^{n-1 / 2}}{\left(1+x^{2}+x^{2}\right)^{n}} d x d y \\
=h_{0 n}(x)+2 \sum_{m=1}^{n-1} h_{m n}(x) \cos 2 m \alpha
\end{gathered}
$$

$$
h_{m n}(x)=\frac{(2 n-2 m-1) !}{(2 n+2 m-1) !} B_{m n} \int_{-1}^{1} 2 P_{2 n-1}^{2 m}\left(\cos \theta_{1}\right) \frac{\cos 2 m \tau}{\left(1+x^{2}+x^{2}\right)^{n}} d x
$$

From (14), (29) and (45) it now follows that (23) is true, the value of $f_{m n}(x)$ being

$$
f_{m n}(x)=g_{m n}(x)+h_{m-1, n}(x)+h_{n n}(x),
$$

where, for $m=n$, we make $h_{n, n}(x)=0$. 
To evaluate (46), we use (25) and the formula $2 \cos 2 m \varphi \cos 2 m \boldsymbol{c}$ $=\cos 2 m(\varphi-\tau)+\cos 2 m(\varphi+\tau)$, and obtain

$2 P_{2 n-1}^{2 m}\left(\cos \theta_{1}\right) \cos 2 m \tau$

$$
\begin{aligned}
=\frac{(-1)^{m}(2 n+2 m-1) !}{(2 n-1) ! 2 \pi} & {\left[\int_{-\pi}^{\pi}\left(\cos \theta_{1}+i \sin \theta_{1} \cos \varphi\right)^{2 n-1} \cos 2 m(\varphi+\tau) d \varphi\right.} \\
+ & \left.\int_{-\pi}^{\pi}\left(\cos \theta_{1}+i \sin \theta_{1} \cos \varphi\right)^{2 n-1} \cos 2 m(\varphi-\tau) d \varphi\right] .
\end{aligned}
$$

Since the integrand has the period $2 \pi$, we may replace $\varphi$ by $\varphi-\tau+\frac{1}{2} \pi$ in the first and by $\varphi+\tau+\frac{1}{2} \pi$ in the second of these integrals without changing the limits of integration, and consequently

$2 P_{2 n-1}^{2 m}\left(\cos \theta_{1}\right) \cos 2 m \tau$

$$
\begin{array}{r}
=\frac{(2 n+2 m-1) !}{(2 n-1) ! 2 \pi}\left[\int_{-\pi}^{\pi}\left(\cos \theta_{1}-i \sin \theta_{1} \sin (\varphi-\tau)\right)^{2 n-1} \cos 2 m \varphi d \varphi\right. \\
\left.+\int_{-\pi}^{\pi}\left(\cos \theta_{1}-i \sin \theta_{1} \sin (\varphi+\tau)\right)^{2 n-1} \cos 2 m \varphi d \varphi\right] .
\end{array}
$$

But from (41) and (28) we find

$$
\begin{aligned}
\cos \theta_{1}-i \sin \theta_{1} \sin (\varphi-\imath) & =\frac{1-i x \sin \varphi+i x \cos \varphi}{\left(1+x^{2}+x^{2}\right)^{1 / 2}} \\
& =(\cos \beta+i \sin \beta \cos \varphi) \sin \theta-i \sin \varphi \cos \theta, \\
\cos \theta_{1}-i \sin \theta_{1} \sin (\varphi+\tau) & =(\cos \beta-i \sin \beta \cos \varphi) \sin \theta-i \sin \varphi \cos \theta,
\end{aligned}
$$

so that, applying the binomial theorem,

$2 P_{2 n-1}^{2 m}\left(\cos \theta_{1}\right) \cos 2 m \tau$

$$
\begin{gathered}
=\frac{(2 n+2 m-1) !}{(2 n-1) ! 2 \pi} \sum_{p=0}^{2 n-1}\left\{\frac{(2 n-1) !(-i)^{p}}{p !(2 n-1-p) !}(\cos \theta)^{p}(\sin \theta)^{2 n-1-p}\right. \\
\quad \times \int_{-\pi}^{\pi}\left[(\cos \beta+i \sin \beta \cos \varphi)^{2 n-1-p}\right. \\
\left.\left.+(\cos \beta-i \sin \beta \cos \varphi)^{2 n-1-p}\right](\sin \varphi)^{p} \cos 2 m \varphi d \varphi\right\} .
\end{gathered}
$$

The integral vanishes when $p$ is odd, as is seen upon replacing $\varphi$ by $-\varphi$, and consequently 
$2 P_{2 n-1}^{2 m}\left(\cos \theta_{1}\right) \cos 2 m \tau$

$$
\begin{gathered}
=\frac{(2 n+2 m-1) !}{2 \pi} \sum_{p=0}^{n-1}\left\{\frac{(-1)^{p}}{(2 p) !(2 n-2 p-1) !}(\cos \theta)^{2 p}(\sin \theta)^{2 n-2 p-1}\right. \\
\quad \times \int_{-\pi}^{\pi}\left[(\cos \hat{\dot{\alpha}}+i \sin \beta \cos \varphi)^{2 n-2 p-1}\right. \\
\left.\left.+(\cos \beta-i \sin \beta \cos \varphi)^{2 n-2 p-1}\right](\sin \varphi)^{2 p} \cos 2 m \varphi d \varphi\right\}
\end{gathered}
$$

Introducing this in (46), and taking $\theta$ as integration variable instead of $x$ we find with the aid of (38)

$$
h_{m n}(x)=B_{m n}(\cos \beta)^{2 n-1} \sum_{p=0}^{n-1} X_{m n p}(\beta) \psi_{n p}(\gamma),
$$

where

$$
\begin{aligned}
X_{m n p}(\beta)= & \frac{(-1)^{p}(2 n-2 m-1) !}{\pi(2 p) !(2 n-2 p-1) !} \int_{-\pi}^{\pi}\left[(\cos \beta+i \sin \beta \cos \varphi)^{2 n-2 p-1}\right. \\
& \left.\quad+(\cos \beta-i \sin \beta \cos \varphi)^{2 n-2 p-1}\right](\sin \varphi)^{2 p} \cos 2 m \varphi d \varphi .
\end{aligned}
$$

We note some special cases of (49). When $p=0$, we have

$$
\begin{aligned}
X_{m n 0}(\beta)=\frac{(2 n-2 m-1) !}{\pi(2 n-1) !} & {\left[\int_{-\pi}^{\pi}(\cos \beta+i \sin \beta \cos \varphi)^{2 n-1} \cos 2 m \varphi d \varphi\right.} \\
& \left.+\int_{-\pi}^{\pi}(\cos \beta-i \sin \beta \cos \varphi)^{2 n-1} \cos 2 m \varphi d \varphi\right],
\end{aligned}
$$

and by (25)

$\int_{-\pi}^{\pi}(\cos \beta+i \sin \beta \cos \varphi)^{2 n-1} \cos 2 m \varphi d \varphi=\frac{(-1)^{m} 2 \pi(2 n-1) !}{(2 n+2 m-1) !} P_{2 n-1}^{2 n}(\cos \beta)$,

which remains unchanged if we change the sign of $i$. Hence

$$
X_{m n 0}(\beta)=\frac{(-1)^{m} 4(2 n-2 m-1) !}{(2 n+2 m-1) !} P_{2 n-1}^{2 m}(\cos \beta) .
$$

Next, let $p=n-1$; then (49) gives

$X_{m, n, n-1}(\beta)=\frac{(-1)^{n-1}(2 n-2 m-1) !}{\pi(2 n-2) !} \int_{-\pi}^{\pi} 2 \cos \beta(\sin \varphi)^{2 n-2} \cos 2 m \varphi d \varphi$, and since the integrand has the period $2 \pi$, we may replace $\varphi$ by $\varphi+\frac{1}{2} \pi$ without changing the limits of integration, so that 
(51) $\int_{-\pi}^{\pi}(\sin \varphi)^{2 n-2} \cos 2 m \varphi d \varphi=(-1)^{m} \int_{-\pi}^{\pi}(\cos \varphi)^{2 n-2} \cos 2 m \varphi d \varphi$, whence, applying (31),

$$
X_{m, n, n-1}(\beta)=\frac{(-1)^{n-m-1}(2 n-2 m-1) !}{2^{2 n-4}(n-m-1) !(n+m-1) !} \cos \beta \text { for } 0 \leqq m \leqq n-1
$$

Finally, for $\beta=0$, (49) becomes

$$
X_{m n p}(0)=\left(\frac{(-1)^{p}(2 n-2 m-1) !}{\pi(2 p) !(2 n-2 p-1) !}\right) 2 \int_{-\pi}^{\pi}(\sin \varphi)^{2 p} \cos 2 m \varphi d \varphi
$$

or by (51) and (31),

$$
\begin{aligned}
X_{m n p}(0) & =\frac{(-1)^{p-m}(2 n-2 m-1) !}{2^{2 p-2}(p-m) !(p+m) !(2 n-2 p-1) !} \quad \text { for } p \geqq m, \\
& =0 \text { for } p<m .
\end{aligned}
$$

In the general case, we may evaluate (49) by means of the well known formula

whence

$$
(\sin \varphi)^{2 p}=\frac{1}{2^{2 p}} \frac{(2 p) !}{p ! p !}+2 \sum_{s=1}^{p}\left(\frac{(-1)^{s}(2 p) !}{(p-s) !(p+s) !} \cos 2 s \varphi\right)
$$

$$
(\sin \varphi)^{2 p} \cos 2 m \varphi=\frac{1}{2^{2 p}}\left[\frac{(2 p) !}{p ! p !} \cos 2 m \varphi\right.
$$

$$
\begin{aligned}
& +\sum_{s=1}^{p}\left(\frac{(-1)^{s}(2 p) !}{(p-s) !(p+s) !} \cos 2(m+s) \varphi\right) \\
& \left.+\sum_{s=1}^{p}\left(\frac{(-1)^{s}(2 p) !}{(p-s) !(p+s) !} \cos 2(m-s) \varphi\right)\right] .
\end{aligned}
$$

Introducing this in (49) and using (25), it is seen that $X_{m n p}(\beta)$ is expressible linearly in the various functions $P_{2 n-2 p-1}^{2 s}(\cos \beta)$, where $s$ takes all the values from 0 to $n-p-1$, the coefficients having a very simple form. For the small values of $m, n$ and $p$ which we need in the next paragraph, it is, however, quite as expedient to evaluate (49) directly, by expanding the binomials and applying (31), in the cases not covered by (50) and (52).

5. Evaluation of the coefficients up to $n=3$ inclusive. From (15) it follows that $\left|R_{3}\right| \leqq\left|\varphi_{4}(\alpha, x)\right| \lambda^{8}+\left|R_{4}\right|$. Evaluating $\varphi_{4}(\alpha, x)$ by the method of the preceding paragraph, and computing its numerical value 
for $\lambda=0.35$ and various values of $\alpha$ and $x$, as well as estimating $\left|R_{4}\right|$ by means of (21), it is found that for $\lambda \leqq 0.35$, we have $\left|R_{3}\right|<0.002$.

To obtain $I / L$ with an error less than two units in the third decimal place, it is therefore sufficient to retain terms containing powers of $\lambda$ not higher than the sixth, so that we only need to calculate $f_{m n}(x)$ for $n=1$, 2 and 3. Writing

we obtain from (39)

$$
\cos \gamma=b,
$$

$$
\begin{aligned}
& \psi_{10}(\gamma)=b, \\
& \psi_{20}(\gamma)=b-\frac{2}{3} b^{3}+\frac{1}{5} b^{5}, \\
& \psi_{21}(\gamma)=\frac{1}{3} b^{3}-\frac{1}{5} b^{5}, \\
& \psi_{30}(\gamma)=b-\frac{4}{3} b^{3}+\frac{6}{5} b^{5}-\frac{4}{7} b^{7}+\frac{1}{9} b^{9}, \\
& \psi_{31}(\gamma)=\quad \frac{1}{3} b^{3}-\frac{3}{5} b^{5}+\frac{3}{7} b^{7}-\frac{1}{9} b^{9}, \\
& \psi_{32}(\gamma)= \\
& \frac{1}{5} b^{5}-\frac{2}{7} b^{7}+\frac{1}{9} b^{9} .
\end{aligned}
$$

The values of $A_{m n}$ and $B_{m n}$ computed from (33) and (44) are

$$
\begin{array}{ll}
A_{11}=2, & \\
A_{12}=1, & A_{22}=30, \\
A_{13}=-21 / 4, & A_{23}=175, \quad A_{33}=1890, \\
B_{01}=2, & \\
B_{02}=1, & B_{12}=10, \\
B_{03}=-7 / 12, & B_{13}=49, \quad B_{23}=378 .
\end{array}
$$

From (49)-(54) we obtain the following values of $X_{m n p}(\beta)$ :

$$
\begin{aligned}
& X_{010}(\beta)=4 \cos \beta \\
& X_{020}(\beta)=-6 \cos \beta+10 \cos ^{3} \beta, \\
& X_{021}(\beta)=-6 \cos \beta, \\
& X_{120}(\beta)=-\frac{1}{2} \cos \beta\left(1-\cos ^{2} \beta\right),
\end{aligned}
$$




$$
\begin{aligned}
& X_{121}(\beta)=\frac{1}{2} \cos \beta, \\
& X_{030}(\beta)=\frac{15}{2} \cos \beta-35 \cos ^{3} \beta+\frac{63}{2} \cos ^{5} \beta \\
& X_{031}(\beta)=15 \cos \beta-35 \cos ^{3} \beta \\
& X_{032}(\beta)=\frac{15}{2} \cos \beta, \\
& X_{130}(\beta)=\left(\frac{1}{4} \cos \beta-\frac{3}{4} \cos ^{3} \beta\right)\left(1-\cos ^{2} \beta\right), \\
& X_{131}(\beta)=\frac{1}{2} \cos \beta, \\
& X_{132}(\beta)=-\frac{1}{4} \cos \beta, \\
& X_{230}(\beta)=\frac{1}{2^{5} 3} \cos \beta\left(1-\cos ^{2} \beta\right)^{2}, \\
& X_{231}(\beta)=-\frac{1}{2^{4}} \cos \beta\left(1-\cos ^{2} \beta\right), \\
& X_{232}(\beta)=\frac{1}{2^{5} 3} \cos \beta .
\end{aligned}
$$

The calculation is now readily completed by means of (40), (48) and (47), and writing

$$
\cos ^{2} \beta=a,
$$

we obtain the results stated in the introductory paragraph.

6. The special case $\alpha=0, x=0$. The result of this paragraph is needed for the computation of the table at the end of paragraph 3 , and, since it is derived by an independent method, it will also serve as a partial check on the numerical values of the coefficient in the general case.

When $\alpha=0$, it is obvious that $I^{\prime}=I^{\prime \prime}$, so that $I=2 I^{\prime}$, and making also $x=0$, it follows from (2), (3) and (4) that

$$
\frac{I}{L}=F(\lambda)+F(-\lambda)
$$

where

$$
F(\lambda)=\int_{-1}^{1} \int_{-1}^{1} \frac{2 \lambda}{\varrho} d x d y
$$

and

$$
\rho^{2}=(1-\lambda)^{2}+(x-\lambda y)^{2}
$$


To evalute (58), we follow Campbell (loc. cit.) in introducing the identity

$$
\frac{\lambda}{\varrho}=\frac{\partial}{\partial x} \frac{\lambda x}{\varrho}+\frac{\partial}{\partial y} \frac{\lambda y}{\varrho}+\frac{\partial^{2} \varrho}{\partial x \partial y},
$$

from which it is seen that

$$
\begin{aligned}
F(\lambda)= & \int_{-1}^{1} \frac{2 \lambda d y}{\left[(1-\lambda)^{2}+(1-\lambda y)^{2}\right]^{1 / 2}}+\int_{-1}^{1} \frac{2 \lambda d y}{\left[(1-\lambda)^{2}+(1+\lambda y)^{2}\right]^{1 / 2}} \\
& +\int_{-1}^{1} \frac{2 \lambda d x}{\left[(1-\lambda)^{2}+(x-\lambda)^{2}\right]^{1 / 2}}+\int_{-1}^{1} \frac{2 \lambda d x}{\left[(1-\lambda)^{2}+(x+\lambda)^{2}\right]^{1 / 2}} \\
& +4 \sqrt{2}(1-\lambda)-4 \sqrt{2\left(1+\lambda^{2}\right)}
\end{aligned}
$$

or evaluating the integrals,

$$
\begin{gathered}
F(\lambda)=4(1+\lambda) \log \left(\frac{1+\lambda+\sqrt{2\left(1+\lambda^{2}\right)}}{(\sqrt{2}+1)(1-\lambda)}\right)+8 \lambda \log (V \overline{2}+1) \\
+4 \sqrt{2}(1-\lambda)-4 \sqrt{2\left(1+\lambda^{2}\right) .}
\end{gathered}
$$

To expand $I / L$ in powers of $\lambda$, we note that

$$
\begin{aligned}
F(\lambda)= & 4 \sqrt{2}(1+\lambda) \int_{0}^{\lambda} \frac{d \lambda}{(1-\lambda)\left(1+\lambda^{2}\right)^{1 / 2}}+8 \lambda \log (\sqrt{2}+1) \\
& +4 \sqrt{2}(1-\lambda)-4 \sqrt{2\left(1+\lambda^{2}\right)}
\end{aligned}
$$

as is readily seen by differentiating the logarithm occurring in (60). It now also follows that

$$
\begin{aligned}
F(-\lambda)= & -4 \sqrt{2}(1-\lambda) \int_{0}^{\lambda} \frac{d \lambda}{(1+\lambda) \overline{\left(1+\lambda^{2}\right)^{1 / 2}}}-8 \lambda \log (\sqrt{2}+1) \\
& +4 \sqrt{2}(1+\lambda)-4 \sqrt{2\left(1+\lambda^{2}\right)},
\end{aligned}
$$

and adding the two expressions,

$$
\begin{aligned}
\frac{I}{L}=8 \sqrt{2}\left[\int_{0}^{\lambda} \frac{\lambda d \lambda}{\left(1-\lambda^{2}\right)\left(1+\lambda^{2}\right)^{1 / 2}}\right. & \\
& \left.+\lambda \int_{0}^{\lambda} \frac{d \lambda}{\left(1-\lambda^{2}\right)\left(1+\lambda^{2}\right)^{1 / 2}}+1-\left(1+\lambda^{2}\right)^{1 / 2}\right] .
\end{aligned}
$$


Introducing the binomial expansions of $\left(1-\lambda^{2}\right)^{-1},\left(1+\lambda^{2}\right)^{-1 / 2}$ and $\left(1+\lambda^{2}\right)^{1 / 2}$, we obtain the desired expansion

$$
\frac{I}{L}=\sum_{n=1}^{\infty} c_{n} \lambda^{2 n},
$$

where

(64)

$$
\begin{aligned}
c_{1}= & 8 \sqrt{2} \\
c_{n}= & 4 \sqrt{2}\left[\left(\frac{1}{n}+\frac{2}{2 n-1}\right)\left(1+\sum_{s=1}^{n-1} \frac{(-1)^{s} \cdot 1 \cdot 3 \cdot 5 \cdots(2 s-1)}{2^{s} \cdot s !}\right)\right. \\
& \left.+\frac{(-1)^{n} \cdot 1 \cdot 3 \cdot 5 \cdots(2 n-3)}{2^{n-1} \cdot n !}\right] . \text { for } n>1 .
\end{aligned}
$$

Computing a few of these coefficients, we find

$$
\begin{aligned}
\frac{I}{L}= & \sqrt{2}\left(8 \lambda^{2}+\frac{10}{3} \lambda^{4}+\frac{31}{3 \cdot 5} \lambda^{6}+\frac{85}{2^{3} \cdot 7} \lambda^{8}+\frac{859}{2^{4} \cdot 3^{2} \cdot 5} \lambda^{10}+\frac{771}{2^{6} \cdot 11} \lambda^{12}\right. \\
& \left.+\frac{10851}{2^{7} \cdot 7 \cdot 13} \lambda^{14}+\frac{3249}{2^{10} \cdot 5} \lambda^{16}+\frac{150065}{2^{12} \cdot 3 \cdot 17} \lambda^{18}+\cdots\right)
\end{aligned}
$$

NEW YoRK, N. Y. 